\title{
Normatização da Concessão do Título de Especialista em Medicina do Esporte e do Reconhecimento de Cursos de Especialização em Medicina do Esporte
}

\author{
Sociedade Brasileira de Medicina do Esporte
}

Revisão 2 - Abril de 2002

Este documento é a segunda revisão da Normatização da Concessão do Título de Especialista em Medicina do Esporte (TEME) e do Reconhecimento dos Cursos de Especialização em Medicina do Esporte (CEME) da Sociedade Brasileira de Medicina do Esporte (SBME).

Comissão para Normatização da Concessão do TEME e Reconhecimento dos CEME 2001-2003:

Presidente: Dr. José Kawazoe Lazzoli (RJ)

Membros: Prof. Dr. Antonio Claudio Lucas da Nóbrega (RJ)

Dr. Arnaldo José Hernandez (SP)

Dr. Marcelo Bichels Leitão (PR)

Dr. Ricardo Munir Nahas (SP)

\section{Comissão do Título de Especialista e Reconhecimento de Cursos de Especialização}

1. A Comissão responsável pelo TEME e reconhecimento dos CEME será composta por 5 (cinco) membros, sendo presidida pelo Diretor Científico de cada nova diretoria eleita e 4 (quatro) outros membros cujos nomes serão indicados pelo presidente da comissão e aprovados pela diretoria. O mandato da comissão tem duração de 2 (dois) anos; seus membros poderão ser reconduzidos uma única vez, sendo obrigatória a renovação de pelo menos um membro a cada nova diretoria.

2. Todos os membros da Comissão deverão possuir Título de Especialista emitido pela SBME/Associação Médica Brasileira (AMB), deverão ser membros efetivos da SBME e não poderão estar coordenando Curso de Especialização em Medicina do Esporte.

3. As funções desta Comissão incluem a condução dos processos de:

a) Elaboração, correção e supervisão da realização das provas para o TEME, pontuação dos curriculum vitae e decisão final sobre a concessão dos TEME;

b) Avaliação e reconhecimento dos Cursos de Especialização em Medicina do Esporte; c) Julgamento dos recursos apresentados por candidatos ao TEME ou por coordenadores de CEME.

d) Indicação das cidades-sede para realização das provas para o TEME;

e) Elaboração dos critérios para revalidação do TEME.

\section{PARTE I - CONCESSÃO DO TÍTULO DE ESPECIALISTA EM MEDICINA DO ESPORTE}

O Título de Especialista em Medicina do Esporte (TEME) emitido pela SBME/AMB identifica o profissional médico com formação acadêmico-científica adequada e apto a exercer a especialidade com ética, responsabilidade e competência. Os critérios aqui estabelecidos estão de acordo com as exigências estabelecidas no convênio firmado pela AMB e Conselho Federal de Medicina em 10/2/1989 e na Normativa de Regulamentação para Obtenção de Título de Especialista ou Certificado de Área de Atuação da AMB, de dezembro de 2001.

1. As provas para concessão do TEME ocorrem uma vez por ano. Nos anos ímpares, durante os Congressos Brasileiros de Medicina do Esporte; nos anos pares, durante eventos da área em âmbito estadual, regional ou nacional. Nestes casos, a Comissão do Título de Especialista da SBME continua responsável por todas as decisões referentes ao processo, mas caberá à organização do evento prover a infra-estrutura local e custear a presença dos membros da Comissão para a Prova Escrita. Os organizadores de eventos que pretendam abrigar a prova para obtenção do TEME nos anos pares, deverão enviar um ofício à Diretoria Científica até 30 (trinta) dias antes do Congresso Brasileiro de Medicina do Esporte a ser realizado no ano precedente. Nesse caso, a cidade-sede será escolhida durante o Congresso Brasileiro de Medicina do Esporte, em reunião da Comissão do TEME.

2. As provas incluem a realização de uma Prova Escrita de caráter eliminatório e uma Avaliação de Títulos (análise do curriculum vitae). 
3. A SBME deverá elaborar um Edital com as normas aqui definidas e demais informações relevantes. O lançamento do Edital deverá ser anunciado no Jornal da AMB e em pelo menos um jornal de grande circulação nacional com antecedência mínima de 60 dias da data da prova. O Edital deverá ser publicado na íntegra na Revista Brasileira de Medicina do Esporte.

\section{I - Documentos necessários para inscrição}

1. Solicitação de inscrição assinada pelo candidato e encaminhada à Diretoria Científica da SBME constando nome completo, endereço residencial, endereço comercial, endereço de $e$-mail, telefones/fax para contato e indicação de uma - e apenas uma - das duas subáreas de interesse (fisiologia/clínica do esporte ou ortopedia/traumatologia do esporte);

2. Cópia do documento de identidade do CRM;

3. Certidão de nada-consta emitida pelo CRM;

4. Curriculum vitae comprovado com documentos adequados;

5. Cheque nominal à SOCIEDADE BRASILEIRA DE MEDICINA DO ESPORTE referente à taxa de inscrição no valor de $\mathrm{R} \$ 200,00$ (duzentos reais).

$\mathrm{OBS}_{1}$ : Os solicitantes que enviarem todos os documentos necessários receberão uma confirmação de inscrição via fax, correio ou e-mail; entretanto, essa confirmação não tem caráter oficial, havendo a possibilidade, por razões diversas, do não recebimento por parte do candidato; assim sendo, o candidato deverá permanecer atento às datas e caso não receba a confirmação - deverá entrar em contato com a Diretoria Científica.

$\mathrm{OBS}_{2}$ : Serão considerados para análise do curriculum vitae somente os documentos enviados no ato da inscrição, não sendo aceita a adição posterior de documentos.

$\mathrm{OBS}_{3}$ : As remessas recebidas que não contiverem um ou mais dos itens acima não serão processadas e desta forma o solicitante não estará inscrito para a prova. Nestes casos, o cheque não será depositado, mas somente será devolvido ao próprio emitente em pessoa ou caso seja fornecido envelope previamente endereçado e selado. A devolução da documentação recebida estará submetida às mesmas condições.

$\mathrm{OBS}_{4}$ : As inscrições que forem enviadas após o prazo estabelecido no Edital (conforme a data do carimbo do correio) não serão processadas e desta forma o solicitante não estará inscrito para a prova; não serão aceitas solicitações de inscrição e cópias de documentos enviadas por fax ou $e$-mail.

$\mathrm{OBS}_{5}$ : A inscrição do candidato pressuporá o conhecimento e a incondicional aceitação das normas e condições estabelecidas no Edital, em relação às quais não poderá alegar desconhecimento.

\section{II - Critérios para concessão do TEME}

1. Ser formado há pelo menos 2 (dois) anos em Faculdade de Medicina reconhecida pelo órgão governamental competente;

2. Estar regularmente inscrito no CRM;

3. Não ter sido condenado em processo ético-profissional no CRM;

4. Atuar profissionalmente e/ou estagiar formalmente na área pelo período mínimo de 1 (um) ano;

5. Obter no mínimo 50\% de acerto na Prova Escrita, ou seja, 30 pontos; o candidato que não atender este critério será eliminado, independentemente da pontuação total;

6. Obter no mínimo $60 \%$ do total de pontos possíveis, que corresponde ao somatório dos pontos da Prova Escrita e da Análise de Títulos.

\section{III - Provas}

O Exame compreenderá duas fases e obedecerá às seguintes disposições:

\section{PROVA ESCRITA}

A Prova Escrita permitirá a totalização de 60 pontos, dos quais 40 pontos correspondem a questões de múltipla escolha ( 25 pontos da parte geral e 15 pontos da parte específica) e 20 pontos correspondem a questões discursivas específicas da subárea escolhida.

A Prova Escrita terá caráter eliminatório. Os candidatos que não obtiverem ao menos $50 \%$ de acerto, ou seja, 30 pontos, serão sumariamente reprovados. Os candidatos que obtiverem pontuação igual ou superior a $50 \%$ (30 pontos) passam à fase seguinte, de Avaliação de Títulos (análise do curriculum vitae).

\section{AVALIAÇÃO DE TÍTULOS}

Para a pontuação da avaliação de títulos (máximo de 40 pontos), existe redundância, isto é, os pontos podem ser obtidos de diferentes formas, sendo até 25 pontos para formação profissional, até 15 pontos para experiência profissional e até 15 pontos para atividades acadêmicas, científicas e administrativas de acordo com a pontuação abaixo. TODOS OS ITENS DIZEM RESPEITO A ATIVIDADES OU TEMAS RELACIONADOS À MEDICINA DO EXERCÍCIO E DO ESPORTE, exceto quando especificado.

\subsection{Formação profissional (até 25 pontos):}

Dentro da especialidade:

2.1.1. Estágios formais $(>100 \mathrm{~h})$

2 pontos

2.1.2. Cursos de aperfeiçoamento organizados e/ou reconhecidos pela SBME, ou organiza- 
dos pelas regionais reconhecidas pela SBME (carga horária igual ou superior a 30 horas)

2.1.3. Curso de Especialização não reconhecido pela SBME (carga horária igual ou superior a 360 horas)

2.1.4. Curso de Especialização reconhecido pela SBME

Em áreas afins:

2.1.5. Estágios formais (> 200h)

2.1.6. Especialização ou residência médica

2.1.7. Mestrado

2.1.8. Doutorado

20 pontos

25 pontos

2 pontos

5 pontos

8 pontos

10 pontos

2.2. Experiência profissional (até 15 pontos):

a. Atuação profissional efetiva na especialidade:

2.2.1. 1 a 5 anos

3 pontos

2.2.2. 5 a 10 anos

5 pontos

2.2.3. mais de 10 anos

8 pontos

b. Participação em delegações desportivas (por participação):

2.2.4. de Federação Estadual

1 ponto

2.2.5. de Confederação Nacional

2 pontos

2.2.6. do Comitê Olímpico Brasileiro

5 pontos

c. Participação em controle de dopagem em eventos ou fora-de-competição (por ano de atuação regular):

2.2.7. natureza local ou estadual

1 ponto

2.2.8. natureza nacional

2 pontos

2.2.9. natureza internacional

2.2.10. no exterior

3 pontos

5 pontos

2.3. Atividades acadêmicas, científicas e administrativas relacionadas à especialidade (até 15 pontos):

a. Publicação de artigos em periódicos indexados, capítulos de livros ou livros (por publicação):

2.3.1. no Brasil

2 pontos

2.3.2. no exterior

4 pontos

b. Apresentação e publicação de resumos em eventos científicos (por evento):

2.3.3. no Brasil

1 ponto

2.3.4. no exterior

2 pontos

c. Participação em órgãos colegiados governamentais, câmaras técnicas profissionais ou conselhos editoriais de periódicos especializados e indexados (por ano):

2.3.5. local ou regional

3 pontos

2.3.6. nacional

4 pontos

2.3.7. internacional

5 pontos

d. Participação como ouvinte em congressos na especialidade, nos últimos 5 anos (por evento, máximo de 2 pontos):

2.3.8. congressos organizados pelas regionais reconhecidas pela SBME 0,5 ponto
2.3.9. Congressos Brasileiros de Medicina do

Esporte 1 ponto

2.3.10. congressos no exterior 1 ponto

e. Participação docente em cursos e congressos (por evento):

2.3.11. aulas em cursos das regionais reconhecidas pela SBME

2.3.12. aulas em cursos de especialização

2.3.13. participações em congressos como convidado

2.3.14. participações em congressos como organizador

2.3.15. participações em congressos no exterior

f. Atividades administrativas:

1 ponto (até 2 pontos)

1 ponto (até 2 pontos)

1 ponto (até 2 pontos)

2 pontos (até 4 pontos)

2 pontos (até 4 pontos)

2.3.16. participação em diretoria de regional reconhecida pela SBME

2 pontos

2.3.17. participação na diretoria da SBME 10 pontos

3. PONTUAÇÃO GERAL E CRITÉRIO PARA APROVAÇÃO

Os candidatos receberão até 60 pontos na prova escrita e poderão receber até 40 pontos na avaliação de títulos (desde que tenham sido aprovados na prova escrita), perfazendo um total máximo possível de 100 pontos. Para aprovação, são necessários um mínimo de 60 pontos.

\section{IV - Dispensados do exame escrito}

Estarão dispensados do exame escrito os profissionais que se encontram nas seguintes situações:

1) Portadores de certificado de curso de especialização em Medicina do Esporte concluído até 1996 inclusive;

2) Médicos formados até 1984 que, mesmo sem possuir certificado de Curso de Especialização em Medicina do Esporte, pratiquem rotineira e continuamente a Medicina do Exercício e do Esporte nos últimos 5 (cinco) anos.

Nestes casos, a concessão do TEME estará condicionada à aprovação na avaliação de títulos, que significa obter no mínimo 30 (trinta) pontos conforme discriminado acima, na parte III, item 2. Para solicitar a avaliação de seus títulos, os candidatos que se enquadram nos dois casos acima deverão enviar para a Diretoria Científica da SBME, a qualquer data, os seguintes documentos:

1. Solicitação de inscrição constando nome completo, endereço residencial, endereço comercial, endereço de $e$ mail, telefones/fax de contato;

2. Cópia do documento de identidade do CRM;

3. Certidão de nada-consta emitida pelo CRM;

4. Curriculum vitae comprovado com documentos adequados; 
5. Cheque nominal à Sociedade Brasileira de Medicina do Esporte referente à taxa de inscrição no valor de $\mathrm{R} \$$ 200,00 (duzentos reais).

\section{V - Conteúdo programático da prova escrita}

1. Fisiologia do exercício: bioenergética, fisiologia muscular esquelética, efeitos agudos e crônicos sobre órgãos e sistemas, princípios da avaliação funcional e prescrição de exercícios;

2. Epidemiologia do exercício: histórico, papel da atividade física na prevenção primária de doenças, relação dose-resposta entre atividade física e redução da morbimortalidade;

3. Avaliação e condutas clínicas direcionadas a indivíduos que participam da prática de atividade física ou de exercícios competitivos: indivíduos aparentemente saudáveis, desportistas e atletas;

4. Avaliação e condutas clínicas direcionadas a indivíduos que participam da prática de atividade física e/ou de exercícios competitivos e fazem parte de grupos especiais: crianças, idosos, mulheres, portadores de doenças crônico-degenerativas (hipertensos, miocardiopatas, coronariopatas, diabéticos, pneumopatas crônicos, nefropatas, portadores de doenças neurológicas, etc.) e portadores de deficiências;

5. Ortopedia e traumatologia desportiva com ênfase nas medidas de prevenção e reabilitação de lesões;

6. Cineantropometria;

7. Doping e controle anti-doping.

\section{VI - Bibliografia sugerida}

- Carvalho T, Nóbrega ACL, Lazzoli JK, Magni JRT, Rezende L, Drummond FA, et al. Posicionamento oficial da Sociedade Brasileira de Medicina do Esporte sobre atividade física e saúde. Rev Bras Med Esporte 1996;2:7981.

- DeLee, Drez (eds.). Orthopaedic Sports Medicine: principles and practice. Philadelphia: WB Saunders, 1994.

- Durstine JL (ed.). ACSM's Exercise management for persons with chronic diseases and disabilities. Champaign: Human Kinetics, 1997.

- Franklin BA (ed.). ACSM's Guidelines for exercise testing and prescription, $6^{\text {th }}$ edition, Philadelphia: Lippincott Williams \& Wilkins, 2000.

- Froelicher VF, Myers JN. Exercise and the heart. $4^{\text {th }}$ edition, Philadelphia: WB Saunders, 2000.

- Ghorayeb N, Barros Neto TL (eds.). O exercício: preparação fisiológica, avaliação médica, aspectos especiais e preventivos. São Paulo: Editora Atheneu, 1999.

- Lasmar NP, Camanho GL, Lasmar RCP (eds.). Medicina do Esporte. Rio de Janeiro: Revinter, 2002.
- Lazzoli JK, Nóbrega AC, Carvalho T, Oliveira MAB, Teixeira JAC, Leitão MB, et al. Posicionamento oficial da Sociedade Brasileira de Medicina do Esporte sobre atividade física e saúde na infância e adolescência. Rev Bras Med Esporte 1998;4:107-9.

- Leitão MB, Lazzoli JK, Oliveira MAB, Nóbrega ACL, Silveira GG, Carvalho T. Posicionamento Oficial da Sociedade Brasileira de Medicina do Esporte sobre atividade física e saúde na mulher. Rev Bras Med Esporte 2000;6: 215-20.

- Nóbrega ACL. Atividade física em cardiologia. In: Mesquita ET (ed.). Coleção livro de bolso de Cardiologia. Volume 1. Rio de Janeiro: Atheneu, 2001.

- Nóbrega ACL, Freitas EV, Oliveira MAB, Leitão MB, Lazzoli JK, Nahas RM, et al. Posicionamento Oficial da Sociedade Brasileira de Medicina do Esporte e da Sociedade Brasileira de Geriatria e Gerontologia sobre atividade física e saúde no idoso. Rev Bras Med Esporte 1999;5: 207-11.

- Pollock ML, Wilmore JH (eds.). Exercise in health and disease: evaluation and prescription for prevention and rehabilitation. $2^{\text {nd }}$ edition, Philadelphia: WB Saunders, 1990.

- Sociedade Brasileira de Medicina do Esporte. I Consenso de Petrópolis: Posicionamento Oficial sobre esporte competitivo em indivíduos acima de 35 anos. Rev Bras Med Esporte 2001;7:83-92.

\section{VII - Recursos de Candidatos Reprovados}

Os candidatos reprovados que desejarem poderão submeter, por escrito, um recurso à Comissão, que decidirá, em caráter irrevogável, dentro do âmbito da SBME, sobre a manutenção ou não do resultado. O prazo para apresentação do recurso é de 72 horas após a divulgação dos resultados.

\section{VIII - Emissão do Título de Especialista em Medici- na do Esporte}

Os candidatos aprovados receberão uma declaração emitida pela SBME constando a data de aprovação, com a qual deverão solicitar a emissão do TEME à AMB através da entidade federada do Estado. A emissão do título pela AMB estará condicionada ao recebimento da taxa de confecção recolhida ao candidato pela SBME e posteriormente repassada à $\mathrm{AMB}$ e pagamento da taxa de requerimento da $\mathrm{AMB}$ recolhida pela federada.

\section{PARTE II - RECONHECIMENTO DE CURSOS DE ESPECIALIZAÇÃO EM MEDICINA DO ESPORTE}

Os Cursos de Especialização em Medicina do Esporte (CEME) têm representado historicamente no Brasil o âmbi- 
to de formação do especialista e, por esta razão, devem atender a critérios mínimos de qualidade. Visando justamente estimular o atendimento destes critérios mínimos de qualidade por parte dos cursos já existentes e de outros que venham a ser criados, a SBME instituiu o reconhecimento dos CEME, que passa a identificar os CEME que formam especialistas com qualidade e com o aval da SBME. Os CEME contemplados com o reconhecimento da SBME receberão um certificado e poderão mencionar tal reconhecimento no material de divulgação.

As solicitações de reconhecimento devem ser encaminhadas à SBME pelo(a) coordenador(a) do curso anexando as seguintes informações:

1. Histórico e objetivos do curso;

2. Ementa, conteúdo programático, carga horária, número de alunos matriculados e sistema de avaliação das disciplinas;

3. Corpo docente, especificando titulação, disciplinas envolvidas e carga horária de cada professor;
4. Documento da Instituição de Ensino Superior responsável acadêmica pelo curso aprovando oficialmente o seu funcionamento.

\section{Critérios para o reconhecimento do CEME:}

1. Apresentar documentação completa;

2. Atender aos critérios estabelecidos pelo Órgão Governamental competente para o funcionamento dos cursos de pós-graduação lato sensu;

3. Possuir carga horária mínima de 360h;

4. Oferecer um mínimo de $20 \%$ da carga horária total em estágios práticos supervisionados;

5. Ministrar temas abrangentes, incluindo obrigatoriamente os seguintes assuntos: fisiologia do exercício; medicina do exercício; traumato-ortopedia aplicada à atividade física; cineantropometria; prescrição de exercício; anti-doping;

6. Pagar taxa de administração no valor de $\mathrm{R} \$ 500,00$ (quinhentos reais).

Petrópolis, 03 de abril de 2002.

\section{Dr. José Kawazoe Lazzoli}

Diretor Científico e Presidente da Comissão do Título de Especialista Sociedade Brasileira de Medicina do Esporte 